Projets

de paysage

\section{Projets de paysage}

Revue scientifique sur la conception et l'aménagement de l'espace

\section{$24 \mid 2021$}

Le paysage au prisme du politique

\title{
Évolution de la pensée de James Corner et son rôle dans l'émergence du Landscape Urbanism
}

The Evolution of James Corner's Thinking and His Role in the Emergence of Landscape Urbanism

Imène Ouali-Bourahla

\section{CpenEdition}

\section{Journals}

Édition électronique

URL : https://journals.openedition.org/paysage/20880

DOI : $10.4000 /$ paysage. 20880

ISSN : 1969-6124

Éditeur :

École nationale supérieure du paysage de Versailles-Marseille, Institut national des sciences appliquées Centre Val de Loire - École de la nature et du paysage, École nationale supérieure d'architecture et de paysage de Bordeaux, École nationale supérieure d'architecture et de paysage de Lille, Agrocampus Angers

\section{Référence électronique}

Imène Ouali-Bourahla, «Évolution de la pensée de James Corner et son rôle dans l'émergence du Landscape Urbanism », Projets de paysage [En ligne], 24 | 2021, mis en ligne le 20 septembre 2021, consulté le 02 octobre 2021. URL : http://journals.openedition.org/paysage/20880 ; DOI : https:// doi.org/10.4000/paysage.20880

Ce document a été généré automatiquement le 2 octobre 2021.

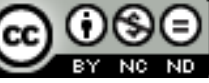

La revue Projets de paysage est mise à disposition selon les termes de la Licence Creative Commons Attribution - Pas d'Utilisation Commerciale - Pas de Modification 4.0 International. 


\title{
Évolution de la pensée de James Corner et son rôle dans l'émergence du Landscape Urbanism
}

\author{
The Evolution of James Corner's Thinking and His Role in the Emergence of \\ Landscape Urbanism
}

Imène Ouali-Bourahla

1 L'impact des théorisations et des pratiques de James Corner sur le champ de l'architecture du paysage commence dès le début des années 1990 (Weller, 2013). Pendant près de 20 ans, il publie plusieurs articles et ouvrages. Ces publications marquent un tournant dans la pensée et la pratique de l'architecture du paysage. Du théorique stricto sensu au plus pragmatique, les textes de Corner reflètent l'évolution de sa pensée dans le temps. Une évolution liée à ses expériences d'enseignement, de recherche et de pratique du projet de paysage. Ses trois premiers articles fournissent un regard critique sur l'architecture du paysage et sa théorisation. Par la suite, Corner s'intéresse au rapport entre représentation et créativité. À travers plusieurs écrits, il met en lumière les enjeux et le rôle de différents modes de représentation sur la créativité des concepteurs et l'imaginaire des habitants. À partir de la fin des années 1990, les textes de James Corner invitent à réfléchir à une nouvelle manière d'appréhender et de concevoir la ville contemporaine. Son ouvrage Recovering the American Landscape, publié en 1999a, reflète cette ambition. Au début des années 2000, il contribue à la construction de la pensée du courant émergent Landscape Urbanism. James Corner est considéré comme l'une des figures emblématiques de ce courant à l'instar des architectes Charles Waldheim et Mohsen Mostafavi.

2 Dès la fin des années 1990, le Landscape Urbanism se définit comme une nouvelle forme de pratique partagée où le paysage tient la place centrale (Corner, 2006). Il «... remplace l'architecture en tant qu'élément constructif de base de l'urbanisme contemporain » (Waldheim, 2006, p.11). Développé aux États-Unis par un groupe d'intellectuels et de professionnels de renom, il représente un courant bien structuré et constitue un discours construit; et ce, comparé à des approches analogues développées 
dans d'autres contextes notamment en France (Donadieu, 2006 ; Leger-Smith, 2014). Ses promoteurs le décrivent comme une approche novatrice inscrite dans la continuité des travaux de Frederick Law Olmsted et de Ian McHarg (Corner, 2006 ; Steiner, 2011). Tandis que d'autres auteurs critiques le considèrent comme une opération de recyclage et de marketing des théories du Landscape Architecture des $\mathrm{XIX}^{\mathrm{e}}$ et $\mathrm{XX}^{\mathrm{e}}$ siècles (Drake, 2010 ; Thompson, 2012 ; Hunt, 2018). Bien que fort intéressant, ce débat autour de la nature et du degré d'innovation (Duany \& al, 2013; Vicenzotti, 2017) du Landscape Urbanism n'est pas l'objet de cet article. L'objectif de ce dernier est d'examiner le rôle que joue James Corner dans l'émergence de ce courant.

Dans un premier temps, nous retraçons le parcours intellectuel et professionnel de James Corner. Pour ce faire, nous analysons le discours de James Corner résultant d'interviews, de conférences, etc. Par cette analyse, nous tentons de saisir ses filiations et ses influences. Le recoupement de ces différentes sources met en évidence de significatives indications. Cette première section permet de saisir les bases et les origines qui structurent sa pensée. Dans un second temps, nous analysons l'ensemble de ses écrits (ouvrages, articles théoriques et articles professionnels) dont une grande partie est publiée dans l'ouvrage The Landscape Imagination: Collected Essays of James Corner (1990-2010) (Corner et Bick Hirsch, 2014). Cette analyse met en lumière la manière dont se construit la pensée de James Corner au fil des années. Et finalement, nous examinons le rôle de cette évolution sur l'émergence du Landscape Urbanism.

\section{James Corner. Figure contemporaine de l'architecture du paysage}

James Corner est né en 1961 à Preston en Angleterre. Il y poursuit toute sa scolarité jusqu'à l'obtention en 1983 de son Degree of Bachelor à l'université de Manchester. En 1984, il intègre le département de Landscape Architecture de l'université de Pennsylvanie où il poursuit deux ans d'études. Il obtient le Certificate of Urban Design en 1985 et le master en Landscape architecture en 1986.

Durant ses deux années d'études à l'université de Pennsylvanie, James Corner suit le programme de l'architecte paysagiste Ian McHarg (1920-2001). Il est passionné par ses cours et ses idées, notamment par son ouvrage Design With Nature publié en 1969 (Corner, 2013). Un ouvrage qui marque une évolution importante, sinon un tournant dans la pratique du projet de paysage. James Corner adhère à la vision de McHarg. Cependant, il trouve qu'elle limite la créativité et l'innovation. Ainsi, sans remettre en cause sa pertinence et son originalité, James Corner s'interroge sur la possibilité de concilier cette approche "rationnelle " avec une dimension poétique et imaginative. Ces questionnements le mènent à s'intéresser à la représentation en tant que source de créativité. Un intérêt qu'il développe aussi bien dans ses travaux d'étudiant que dans ses écrits ultérieurs.

6 À l'université, Corner est considéré comme «trop expérimental et avant-gardiste » (Corner, 2013, p. 156). Pour son Certificate of Urban Design, il étudie le projet de régénération urbaine du quartier en déclin de l'Isle of Dogs à Londres. Dans son analyse, il insiste sur l'importance de mettre en place une approche cohérente et imaginative de la pratique de la conception urbaine. En 1986, pour son mémoire de master, James Corner propose une nouvelle vision pour la ville de Philadelphie 
(figure 1). Le plan qu'il dessine repense la ville par le paysage. Considéré comme novateur, ce projet est distingué par l'American Society of Landscape Architecture (ASLA) Award ${ }^{1}$.

Figure 1. Philadelphie repensée par James Corner (1986)

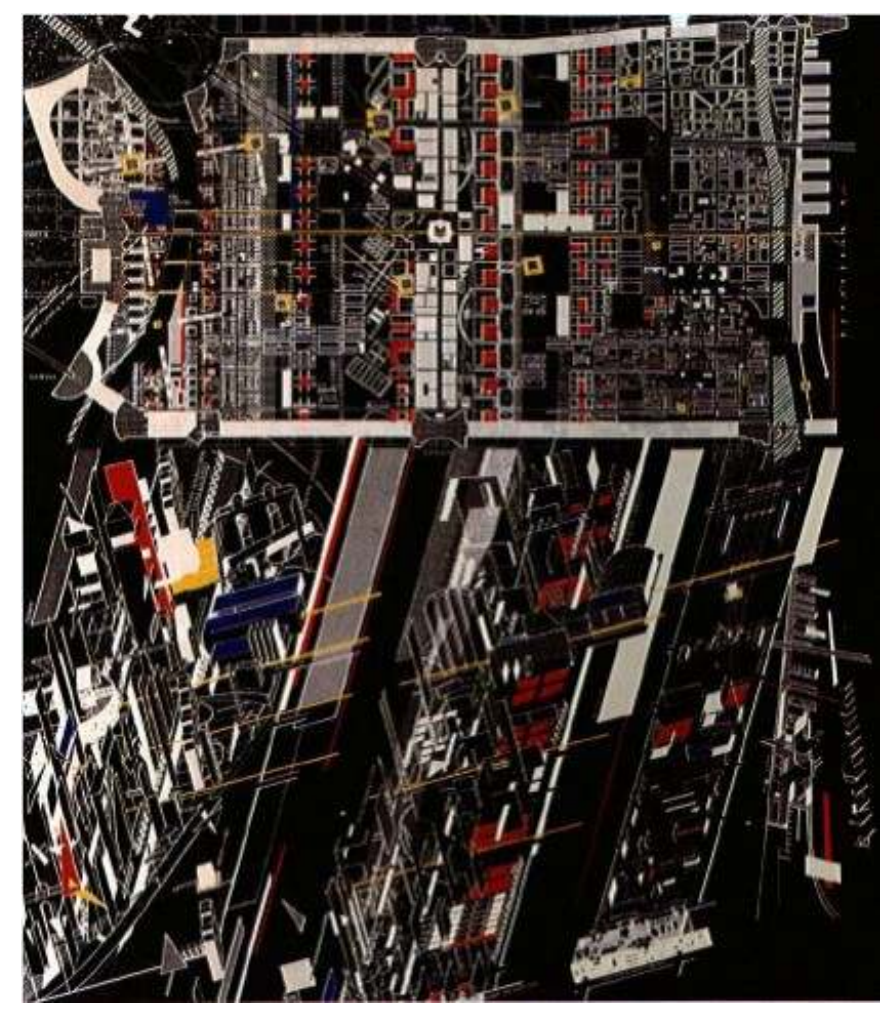

Source : extrait de Landscape Imagination. Collected Essays of James Corner 1990-2010 (Corner et Bick Hirsch, 2014).

\section{Parcours de James Corner. Entre théorie et pratique}

Dans le parcours de James Corner, trois périodes peuvent être observées. La première remonte au début des années 1980 . Elle correspond à sa première expérience en tant qu'étudiant en agence de paysage. La seconde période commence à la fin des années 1980. Après l'obtention de son diplôme de master, Corner entame une carrière de praticien et d'universitaire. Il travaille dans différentes agences de paysage tout en enseignant à l'université de Pennsylvanie. À partir des années 1990, le parcours de James Corner est à la fois théorique et expérimental. Il propose, à travers plusieurs écrits théoriques, des réponses aux problématiques soulevées au cours des deux précédentes périodes. Il considère l'écriture comme une partie intégrante de la pratique du projet tout comme le dessin et la construction (Corner, 2014). En plus de la théorisation, il expérimente ses idées en studio dans le cadre de ses activités d'enseignement et en agence à travers sa pratique professionnelle. 


\section{Premières expériences de terrain}

8 Au début des années 1980, alors qu'il est encore étudiant à Manchester, James Corner travaille en tant que Landscape Architectural Assistant au sein de l'agence londonienne William Gillespie + Partners (1982-1984). Une agence qui travaillait, à ce moment-là, sur plusieurs projets de régénération urbaine. Pendant la préparation de son master, il travaille comme paysagiste au sein de l'agence Robert Fleming Associates (1984-1986). Fondée en 1984 à Philadelphie, cette agence de paysage est spécialisée dans les questions de préservation et de restauration des paysages historiques et naturels. Après l'obtention de son diplôme, il rejoint, en 1987, l'agence Wallace, Roberts \& Todd, une agence $^{2}$ cofondée par Ian McHarg en 1962 à Philadelphie.

\section{Entre pratique et enseignement}

9 À partir de la fin des années 1980, James Corner conjugue pratique en agence et enseignement à l'université. Tout en continuant à travailler chez Wallace, Roberts \& Todd, il est engagé par Anne Whiston Spirn ${ }^{3}$ pour assurer des cours de dessin et de représentation et animer des ateliers de projet (1988-2012) à l'université de Pennsylvanie. En outre, il intervient dans plusieurs autres institutions à l'instar de l'université de Harvard (1993), l'université de l'Illinois (1997) et l'université de Yale (1997).

\section{De la théorisation et de la pratique}

10 Pendant les années 1990, James Corner publie une dizaine d'articles théoriques. Ces derniers s'intéressent à la théorie du design, aux enjeux de la représentation et enfin au renouvellement des paradigmes des projets de paysage.

11 À la fin des années 1990, il fonde son agence Field Operations à New York. L'appellation choisie caractérise et reflète la vision de James Corner du projet. Une vision qui accorde beaucoup d'importance aux forces et dynamiques qui opèrent dans le champ urbain (Nawre et Sen, 2011). L'ascension de Corner dans le milieu professionnel aux USA débute à cette période. Dès les premières années d'existence de Field Operations, ses projets se distinguent par leur démarche, leur approche et leurs modes de représentation. Ils ouvrent de nouvelles façons de faire comme le démontrent la proposition de la Flatbed ${ }^{4}$ (figure 2) en 1998, dans le cadre du projet d'extension de la commune de Älvsjö dans la banlieue de Stockholm (Bélanger, 2015), et la manière d'appréhender les processus écologiques et leur représentation dans les projets de requalification en parc de l'ancienne base aérienne militaire de Downsview à Toronto, en 2000, et de la décharge de Fresh Kills à New York, en 2001 (Waldheim, 2006) (figures 3 et 4 ).

12 Enfin, les années 2000 marquent une évolution dans la nature, la qualité et le nombre des publications de Corner. Focalisés sur sa pratique, ses textes sont plus succincts, plus illustratifs et moins nombreux comparativement aux écrits des années 1990. Ils sont publiés essentiellement dans des revues professionnelles, comme Topos. 
Figure 2. The Älvsjö Flatbed

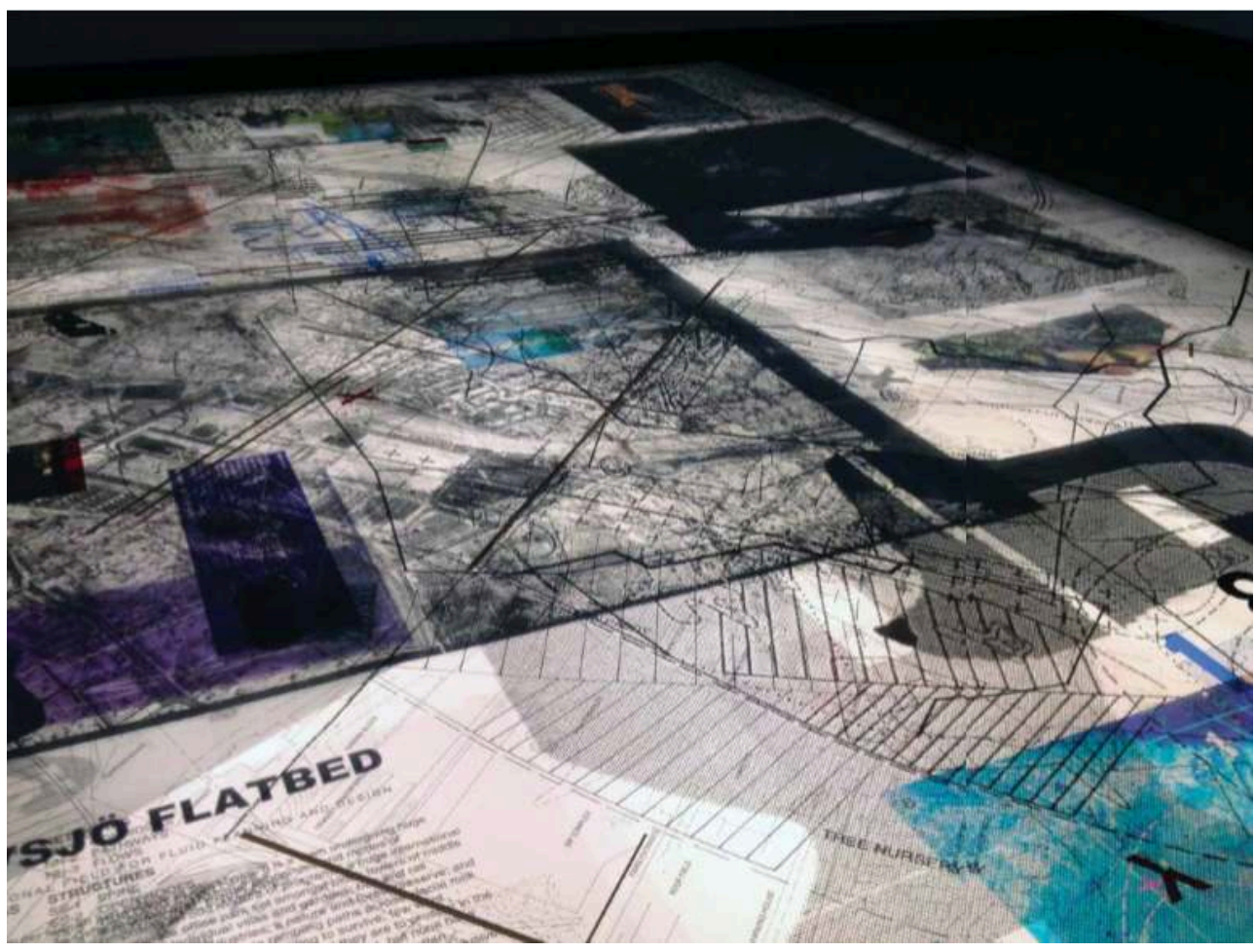

Source : extrait de «Everything on the Table. Reconvering and Reprojeting James Corner's Lost Map » (Bélanger, 2015).

Figure 3. Downsview Park

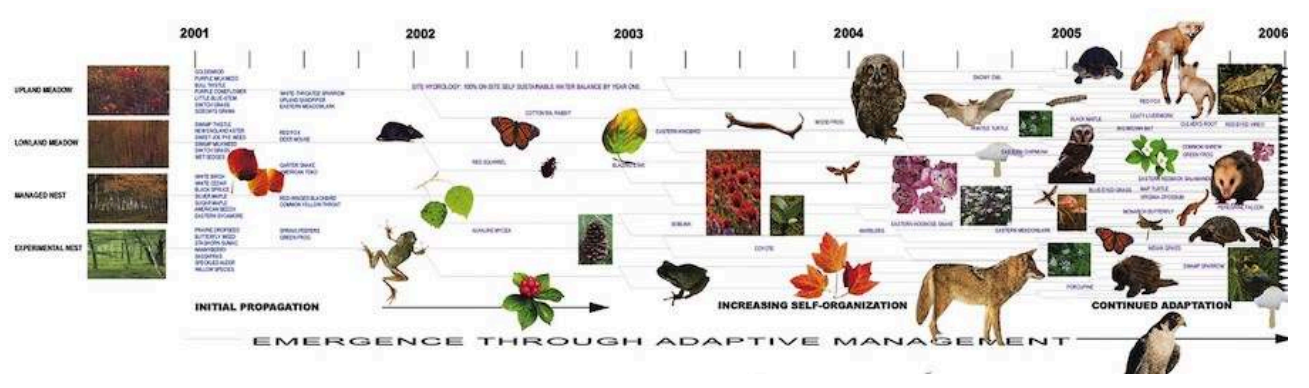

Évolution de la faune et de la flore à travers le temps.

Source : Field Operations, 2001. 
Figure 4. FreshKills Park
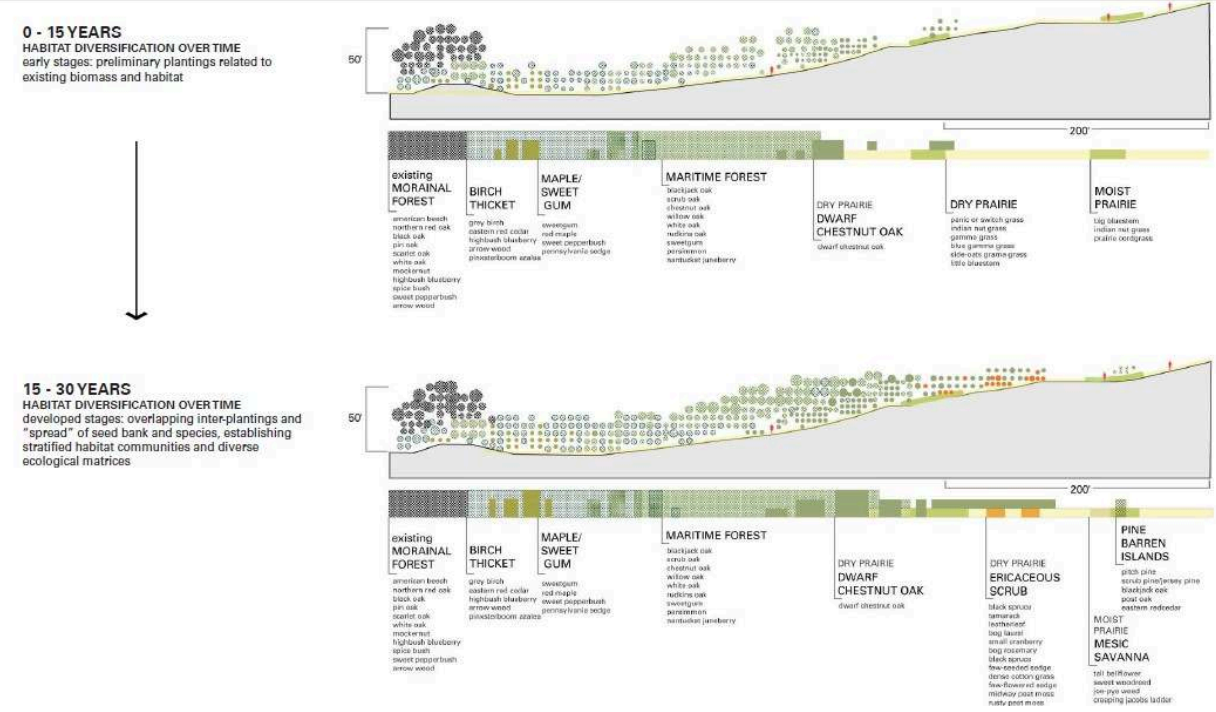

Diversification des habitats au fil du temps.

Source : Field Operations, 2006

\section{Influences et références de James Corner}

Dans une interview menée en 2017, par des étudiants de la Graduate School of Design de Harvard, James Corner indique que dans sa jeunesse, il avait une double sensibilité à la fois environnementale et artistique. Suivre des études d'architecture de paysage était une manière de concilier ces deux centres d'intérêt. Après l'obtention de son Bachelor Degree, il intègre l'université de Pennsylvanie spécialement pour suivre le programme de master de Ian McHarg. À l'époque, il est impressionné par l'Ecological Method de ce dernier, une méthode analytique et projectuelle, développée à la fin des années 1960, pour appréhender les territoires à grande échelle au prisme des processus naturels (figure 5).

La façon de voir et de faire le projet de paysage de James Corner est principalement influencée par Ian McHarg. Dans l'enseignement de ce dernier (1984-1986) mais aussi dans l'étude approfondie de l'ouvrage Design With Nature (figure 6), James Corner trouve des réponses à ses préoccupations, notamment environnementales. Néanmoins, dans un esprit critique, il interroge les limites de l'approche de McHarg qu'il considère comme trop rationnelle. Son premier article, «A Discourse on Theory I; Sounding the Depths. Origins, Theory and Representation " publié en 1990, est représentatif de sa réaction à la rationalité de la méthode de McHarg. Ainsi, tout au long de son parcours, Corner développe ses idées dans une logique «d'équilibre [...] entre l'imagination poétique [...] et les performances écologiques en tant que médium instrumental» (Corner et Bick Hirsch, 2014, p. 15).

«[...] As I then became immersed in the Masters program, McHarg continued to astound with his larger-than-life presence, passionate energy and vision. I must have re-read "Design with Nature" five or six times in that first year! McHarg had inaugurated a whole new landscape project, one that broached all scales and disciplines. » (Corner, 2013. p. 156.) 
Figure 5. Ecological Method, l'analyse en strate

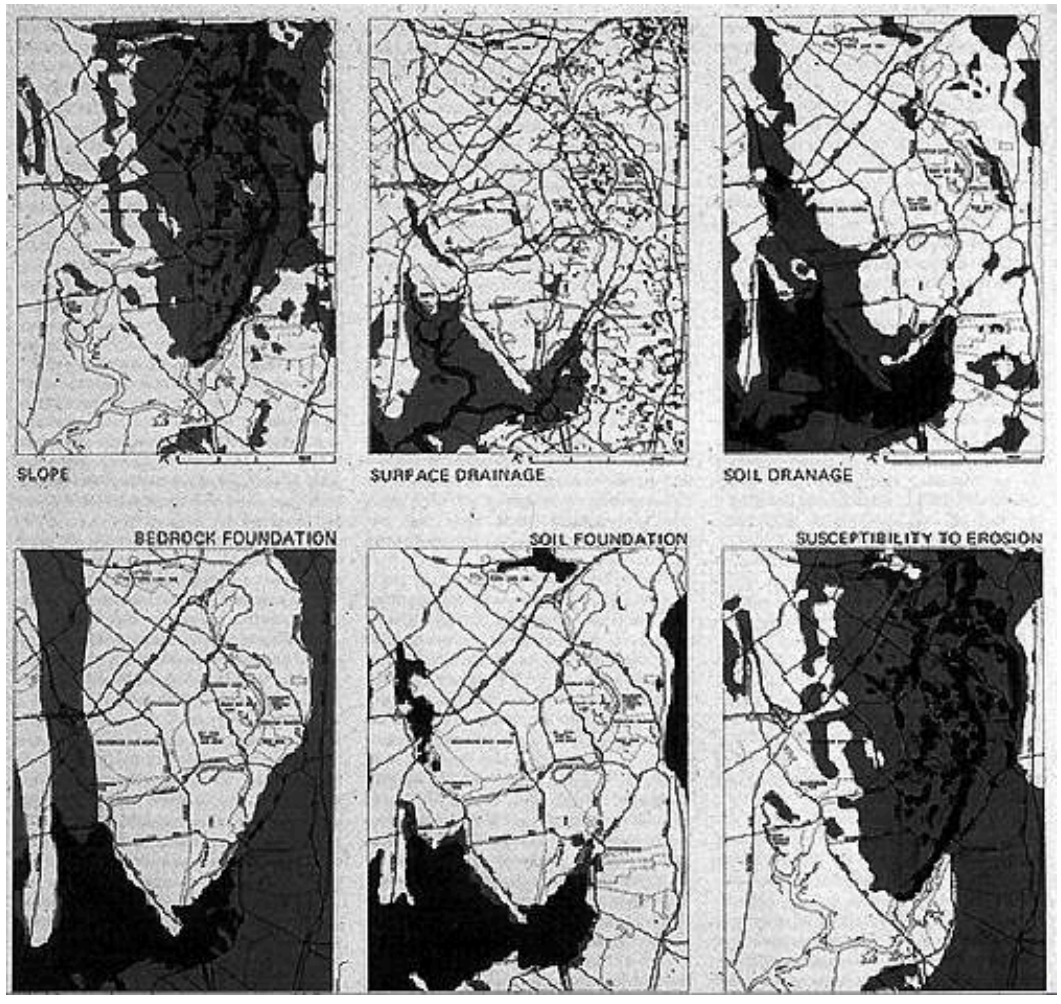

Source : Extrait de Design With Nature (McHarg, 1969).

Figure 6. Exposition « Design with Nature Now », Weitzman School of Design, université de Pennsylvanie, juin 2019

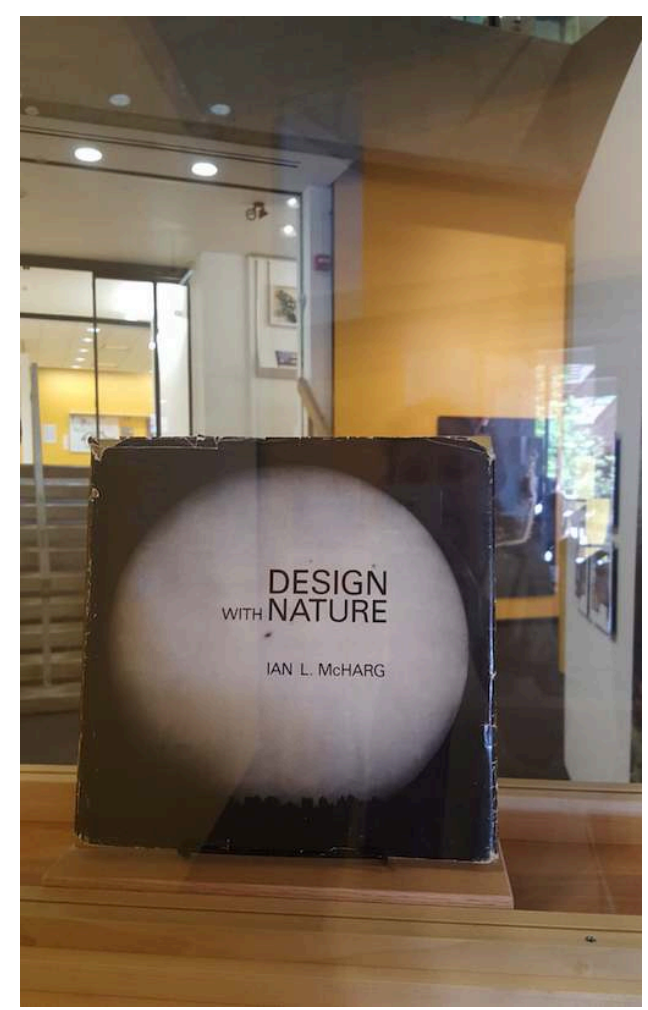

Source : Imène Ouali-Bourahla. 
Dans l'évolution de sa pensée, James Corner se réfère souvent aux travaux des architectes paysagistes américains Frederick Law Olmsted (1822-1903) et Jens Jensen (1860-1951), ce qui témoigne de l'influence de ces deux figures sur sa conception du rapport ville/paysage. Le projet d'Emerlade Necklace à Boston (figure 7) d'olmsted ouvre un nouveau regard sur la relation qu'entretiennent la forme urbaine et les processus environnementaux. Les parcs, promenades, espaces ouverts... sont conçus comme des structures pour le développement urbain et des infrastructures assurant des fonctions de continuité écologique. Le concept de Fitting Design de Jens Jensen s'inscrit également dans cette conception à différentes échelles, des parcs urbains aux villes et territoires. À travers ce concept, Jensen propose une approche intégrant les caractères des systèmes naturels. Cela s'opère notamment à travers l'utilisation des plantes et la prise en compte des dynamiques écologiques des milieux dans les parcs.

Un autre travail plus contemporain constitue une des principales sources d'influence de Corner. Il s'agit du projet du parc de La Villette de Bernard Tschumi (figure 8). Selon Corner, ce projet a contribué à la construction de sa conception du projet urbain en général et du parc en particulier. Alors qu'il était encore étudiant au moment du lancement du projet, Corner prend conscience de l'importance du programme au-delà de la forme et des styles (pastoral, moderne...).

Pendant ses études à l'université de Pennsylvanie, James Corner découvre le Land art à travers les travaux de plusieurs artistes dont Robert Smithson et Michael Heizer. Selon Corner, ces travaux ouvrent sur un nouveau rapport art/nature. Ils permettent de prendre conscience de la dynamique du paysage. Dans ces œuvres «le paysage est moins une scène de contemplation, il est dynamique, terrain de processus naturels induits par le mouvement et le temps » (Corner, 1999a, p. 15).

Figure 7. Frederick Law Olmsted, Emerlade Necklace, Boston, 1894

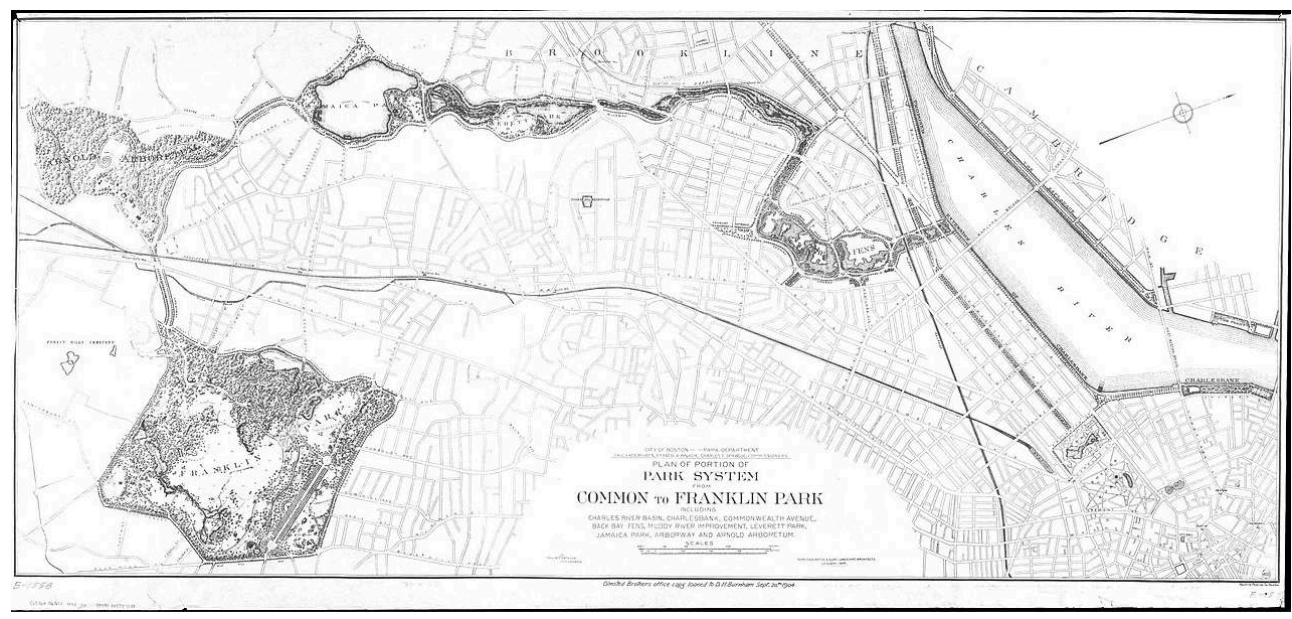

Inscription du parc à différentes échelles.

Source : domaine public. 


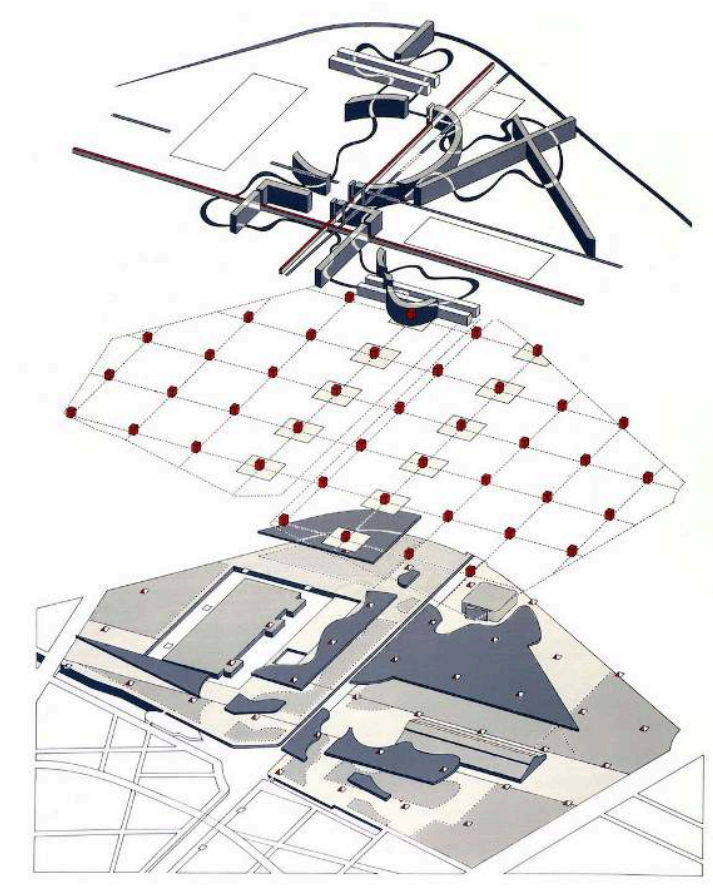

\section{Pensée de James Corner et son évolution dans le temps}

L'ensemble des écrits de James Corner peuvent être scindés en quatre phases thématiques principales. Ce phasage s'appuie sur celui mis en évidence et attesté par plusieurs auteurs qui ont travaillé sur le cadre théorique de James Corner, notamment Alison Bick Hirsch, architecte paysagiste et enseignante à l'université de Caroline du Sud, qui aborde cette question dans l'introduction de l'ouvrage The Landscape Imagination: Collected Essays of James Corner (1990-2010), publié en 2014. Cet ouvrage, coédité avec James Corner, regroupe la majorité des textes de ce dernier.

Au début des années 1990, il commence à écrire en s'intéressant à la théorie de l'architecture du paysage. Ensuite, il analyse les enjeux de l'usage de différents modes de représentation (photographie aérienne, photomontage, cartographie...) dans le champ du projet de paysage. Au début des années 2000 , il contribue à la définition du Landscape Urbanism. Il propose un premier modèle opérationnel en 2003 qu'il réajuste en 2006. À cette période, sa pratique en agence est en plein essor, ce qui se constate dans ses écrits. C'est sur sa pratique que se focalisent les articles publiés dans les années 2000. Différents projets sont abordés pour expliciter l'approche de l'agence Field Operations.

Au-delà des thématiques majeures identifiées, ces textes sont interreliés et leur lecture met en évidence une certaine continuité. Certains articles sont à l'interface de deux thématiques à l'instar de l'article « Field Operations » (1999b) qui aborde l'enjeu de la 
représentation et ce en s'appuyant sur la pratique de projet de l'agence Field Operations.

\section{Théorie de l'architecture du paysage. Vers la mise en place de bases théoriques (1990-1991)}

21 Les premiers écrits de James Corner s'inscrivent dans un contexte marqué par la crise économique mondiale (1970-1990) et la montée des préoccupations environnementales. L'avènement du club de Rome ${ }^{5}$ en 1968, la tenue de la conférence de Stockholm en 1972 et la publication du rapport de Brundtland en 1987 représentent des événements majeurs qui interpellent les sociétés sur la nécessité de changer de paradigme. Pour répondre aux défis environnementaux et socioéconomiques contemporains, Corner estime qu'il est impératif d'associer la théorie à la pratique.

Contrairement à l'architecture, l'architecture du paysage, à cette période, n'est pas construite en tant que discipline. Cette situation est soulignée par plusieurs paysagistes, notamment au cours du Council of Educators in Landscape Architecture (CELA) conférence organisée en 1990 aux USA, qui a interrogé les rôles de la théorie et de la pensée critique dans l'enseignement et la pratique du projet de paysage.

S'inspirant des discours renouvelés de la critique moderne, Corner développe sa vision de la théorie de l'architecture du paysage à travers trois articles, à savoir : "Sounding the Depths - Origins, Theory and Representation» en 1990, "Three Tyrannies of Contemporary Theory and Alternative of Hermeneutics " et "Critical Thinking and Landscape Architecture » en 1991. À travers ces écrits, Corner tente de contribuer à la mise en place de bases théoriques pour une architecture du paysage renouvelée dépassant le présent et se référant au passé.

Dans son premier texte " Sounding the Depths - Origins, Theory and Representation ", Corner interroge le rôle de la théorie dans le champ de l'architecture du paysage. Il associe d'ailleurs la crise environnementale de la fin du $\mathrm{xx}^{\mathrm{e}}$ siècle à une rupture épistémologique avec la tradition. Il considère que cette crise environnementale est due à une vision réductrice du monde naturel. Il explique qu'initialement, dans leur définition, l'art et l'architecture associaient techne (savoirs) et poesis (créativité, représentation). Dès la fin $d u x_{\text {III }}$ siècle, la révolution de la pensée scientifique implique un changement dans cette définition. Les sciences modernes introduisent une séparation entre l'objectif et le subjectif, techne et poesis, ce qui modifie la nature du rôle de la théorie dans la production architecturale (Corner, 1990).

James Corner estime que les différentes approches développées au $\mathrm{xx}^{\mathrm{e}}$ siècle s'inscrivent dans la continuité de cette séparation de l'art, de l'esthétique, de l'écologie et de l'histoire. Dans le cadre de l'enseignement et de la pratique de l'architecture du paysage, il distingue deux courants opposés. D'une part, un courant, de pensée technique, centré sur la résolution de problèmes et le management écologique et, d'autre part, un courant adoptant une approche esthétique qui se base sur des doctrines formalistes ou des considérations historiques. Opposé à cette dichotomie, Corner voit l'architecture du paysage comme un médium, entre nature et culture. Un médium qui rétablit les rapports homme/environnement (Terre) et qui structure les formes d'expériences et de valeurs communes. Dans ce sens, la théorie de l'architecture de paysage devrait, selon Corner, se baser sur les domaines de la perception, de la phénoménologie et de l'imagination. 
26 À travers son enseignement et sa pratique, Corner met l'accent sur l'enjeu de la pensée critique. Cette dernière est considérée comme fondamentale dans la lisibilité et le sens de la conception paysagère. Elle permet, d'une part, de formuler une réponse originale et, d'autre part, d'évaluer et d'apprécier son devenir ou son vécu. Dans son article "Critical Thinking and Landscape Architecture », Corner définit la pensée critique dans la conception paysagère comme un lien entre la théorie et la pratique, entre l'héritage passé et le potentiel futur mais aussi entre l'existant et l'invention. Cette pensée est incarnée dans la forme construite et elle est enrichie par la représentation et le discours, ce que James Corner désigne par l'expression Landscape Imagination. Les travaux de Lewis Mumford, de Frederick Law Olmsted et de Robert Smithson, entre autres, démontrent, selon Corner, les enjeux et la portée de cette pensée critique. Le paysagiste est à la fois critique, stratège, médiateur et " créateur » (Corner, 1991 dans Corner et Bick Hirsch, 2014. p. 45). Sa pratique doit aller au-delà des dualités simplistes art/science, théorie/pratique, nature/culture, construit/imaginaire, etc.

Dans cette série de textes, Corner aborde, en outre, les origines et l'enjeu de la théorie en architecture du paysage. Il défend un modèle théorique plus poétique, qu'il définit en réaction à la vision technoscientifique moderne et à la pensée contemporaine. Dans son article «Three Tyrannies of Contemporary Theory and Alternatuve of Hermeneutics ", il met en évidence les limites de ces deux modes de pensée et préconise l'adoption d'une approche herméneutique. Il considère que cette dernière constitue une alternative aux trois approches prédominantes de la théorie contemporaine. La première approche est positiviste. Dogmatique et empirique, elle se base sur l'analyse factuelle des phénomènes et des processus. L'Ecological Method de Ian McHarg s'inscrit dans cette approche. La deuxième approche utilise des paradigmes et des modèles universels (types). Des méthodes prédéfinies sont adoptées pour la résolution des problèmes. Tandis que la troisième est l'approche des avant-gardes. Elle marque, dans une démarche novatrice ou provocatrice, une rupture avec les pratiques traditionnelles. Contrairement à ces trois approches qui découlent, selon Corner, de la pensée technoscientifique moderne impliquant le contrôle et la domination, l'herméneutique est une pratique interprétative et circonstancielle qui se base sur la perception et les processus culturels. Adopter une approche herméneutique dans la théorie de l'architecture du paysage induit un impact sur le Landscape Imagination, ce qui ouvre sur de nouvelles relations art/science, théorie/pratique, et homme/nature.

\section{Représentation et créativité. Le rôle des modes de représentation dans le projet de paysage (1992-1999)}

La deuxième série de textes aborde l'enjeu de la représentation dans le processus créatif du projet de paysage. Ces textes sont écrits dans une période marquée par l'évolution des considérations environnementales notamment l'avènement du développement durable. À la fin des années 1980, une inflexion durable s'opère pour considérer les activités humaines comme partie intégrante des facteurs ayant une incidence sur les systèmes écologiques, leurs ressources et leurs dynamiques.

Dans le cadre de cette nouvelle vision de la relation homme/nature, Corner présente de nouvelles méthodes de travail reconsidérant la position de l'homme dans le processus du projet. Se basant sur des pratiques de visualisation et de construction, il considère l'usage des différents modes de représentation, dont le digital, comme un eidetic medium 
- image mentale aussi bien picturale que tactile, cognitive, acoustique ou intuitive (Corner, 1999c) - nécessaire dans la construction et la compréhension de l'imaginaire des projets.

Dans son premier article sur ce sujet, «Représentation and Landscape. Drawing and Making in the Landscape Medium » (1992), Corner interroge le rôle du dessin dans les projets. Il considère le dessin comme un moyen de réflexion, un outil de créativité mais aussi un catalyseur d'idées. En tant qu'intermédiaire, le dessin permet d'expliciter le processus de conception en mettant en exergue le lien entre une réalité donnée et une idée mais aussi entre une idée et sa représentation visuelle. Au regard de la dynamique des paysages, Corner explique que le dessin ne doit pas être uniquement analytique à travers une projection de ce qui est construit mais qu'il doit être stratégique, métaphorique et imaginatif. Cela évite l'écart entre ce qui est représenté et ce qui est construit.

31 Après cet article, qui est considéré, de par sa portée, comme un texte fondamental dans l'enseignement de l'architecture du paysage aux USA (Bick Hirsch, 2014), Corner développe son idée en abordant l'intérêt et le rôle des différents modes de représentation en tant que médiums à la fois dans la perception des paysages mais aussi dans l'imaginaire des projets.

En 1995, James Corner et Alex MacLean, connu pour ses photographies aériennes, organisent une exposition intitulée "Taking Measures across the American Landscapes ». Un an après cette exposition, un ouvrage collectif éponyme est publié (Corner, MacLean, Van Valkenburgh, 1996). À travers des textes descriptifs, des photos aériennes, des cartes et des collages de fragments de cartes et de photos, cette exposition aborde la possibilité d'ouvrir le champ de l'architecture du paysage à une échelle plus large pouvant conduire à l'invention de nouveaux paysages (Beard, 1995). Elle interroge l'évolution des paysages culturels "undesigned" (Van Valkenburgh, 1996), en l'occurrence agricoles et technologiques. Pour Corner, ces paysages peuvent constituer une référence pour la création de nouveaux paysages. L'impact de ce travail sur le projet de paysage peut être assimilé à l'impact de la réflexion de Le Corbusier relative aux élévateurs à grain américain sur l'architecture moderne (ibid.).

Dans l'ouvrage Taking Measures across the American Landscapes (1996), Corner analyse la mesure et son potentiel en tant qu'agents réconciliant la représentation symbolique et la technologie moderne. Il démontre dans quelle mesure les paysages conçus peuvent devenir une représentation des paysages culturels "undesigned», qui n'est pas seulement visuelle et descriptive mais qui permet de renseigner comment la population perçoit, façonne et transforme le sol.

Dans la continuité de cette logique de recherche de nouvelles méthodes de projets, Corner s'intéresse à l'écologie en tant qu' « Agent of Creativity» (Corner, 1997) - une source pour la création de nouveaux paysages. La dynamique des paysages et les processus naturels deviennent des sources pour la genèse des concepts des projets. Dans son article "Ecology and Landscape as Agents of Creativity" (1997), Corner aborde l'enjeu de l'appropriation de l'écologie dans la pratique de l'architecture du paysage. Un enjeu qui va au-delà des objectifs de restauration et de protection environnementale revendiqués par les paysagistes américains des xixe et xxe siècles, dont figurent Jens Jensen et Ian McHarg. En effet, Jensen se base sur l'écologie pour reconstituer des paysages et des écosystèmes présents localement. Quant à McHarg, l'écologie, par ses méthodologies et ses données, lui permet de fonder une évaluation 
qualitative et quantitative. Pour Corner, l'écologie s'apparente plutôt à une construction sociale qui peut apporter des informations et initier l'imagination dont découlent les visions et les relations de l'homme au monde naturel. Tout comme le paysage, l'écologie est considérée comme un médium culturel et de représentation.

L'article «Eidetic Operations and New Landscapes » (1999c) aborde le rôle subliminal des différents modes de représentation dont le collage. Corner favorise, entre autres, l'usage du collage dans la pratique du projet de paysage pour son caractère " provocateur » incitant l'observateur à imaginer de nouvelles conditions paysagères. À travers la juxtaposition d'éléments a priori sans rapport, le collage constitue un outil de création d'images eidétiques. Corner explique que l'enjeu de la représentation n'est pas tant dans le type d'images produites que dans la pensée créative et l'imaginaire que ses dernières génèrent. Dans une logique de conception de nouveaux paysages, différents modes de représentation existants peuvent être utilisés et/ou réadaptés pour leur portée eidétique, à l'instar du dessin, du photomontage, de la cartographie, de la modélisation, etc. L'objectif est donc de «imaging» et non de "picturing». Ainsi, les outils du planning et de la création d'images sont utilisés pour appréhender la grande échelle (Corner, 1999b). La cartographie est présentée dans son article « The Agency of Mapping. Speculation, Critique and Invention » (1999d) comme un moyen permettant d'associer le « mesuré » et l'abstrait.

\section{Vers la définition d'un modèle opérationnel. Le Landscape Urbanism (2001-2004)}

La relation ville/paysage a évolué au fil des années. Cette évolution est induite par les changements de considérations vis-à-vis de la ville (sacrée, profane, lieu de divers maux...) et du paysage (médiateur entre ville/territoire, antidote aux pressions urbaines...). Dissocié de la ville au cours du XIX ${ }^{e}$ siècle, le paysage, en tant que notion et outil, est réinvesti, dès la fin $\mathrm{du} \mathrm{xx}^{\mathrm{e}}$ siècle, par les théoriciens et les professionnels de la ville. Ce regain d'intérêt est observé dans plusieurs contextes, discours et approches mettant le paysage au cœur de toute réflexion sur la ville et/ou le territoire.

À la fin des années 1990, aux USA, dans un contexte de désindustrialisation, plusieurs théoriciens et praticiens du paysage plaident en faveur de la mise en place d'une façon différente de voir et de faire la ville. Ainsi, une nouvelle pratique émerge. Appelée Landscape Urbanism, elle est encadrée par plusieurs personnalités de renom du champ de l'architecture du paysage. En avril 1997, Charles Waldheim organise une rencontre, "The Landscape Urbanism Symposium and Exhibition", à la Graham Foundation à Chicago qui, depuis sa création en 1956, soutient les projets qui explorent de nouvelles perspectives pour le développement des pratiques spatiales. Cet événement a rassemblé plusieurs théoriciens de l'architecture du paysage dont James Corner.

À l'instar de Charles Waldheim et de Mohsen Mostafavi, James Corner représente l'une des figures emblématiques à l'origine de l'émergence et de la définition du Landscape Urbanism. Alliant deux termes à la fois opposés et complémentaires, à savoir le paysage et l'urbanisme, le Landscape Urbanism constitue « bien plus qu'une image, un style : c'est une philosophie, une attitude, un mode de penser et d'action » (Corner, 2003a, p. 58). Ce « réalignement disciplinaire " (Waldheim, 2006, p. 37) suggère la reconsidération des méthodes de conception traditionnelles. Il implique le remplacement de l'architecture par le paysage. Ainsi, le paysage devient un modèle d'urbanisme, voire plus un modèle 
de processus. Dans son article «Landscape Urbanism » publié en 2003a dans l'ouvrage Landscape Urbanism. A Manual for the Machinic Landscape de Mohsen Mostafavi, James Corner propose un premier modèle opérationnel, qu'il développe ensuite dans son second article sur le sujet, « Terra Fluxus ", publié en 2006 dans l'ouvrage The Landscape Urbanism Reader de Charles Waldheim.

Pour ouvrir sur un nouveau champ des possibles, Corner s'appuie dans ses articles sur sa pratique de projet. Son article «Field Operations" (Corner, 1999b) porte essentiellement sur l'approche stratégique de son agence nouvellement créée. À travers plusieurs de ses projets, il décrit les possibilités qu'offrent le jeu d'échelles et les processus écologiques, culturels, politiques et économiques. En 2001, il publie deux autres articles intitulés « Downsview Park, Toronto - Emergent Ecologies » (2001a) et «Landscraping" (2001b). Le premier porte sur sa proposition dans le cadre du concours de Downsview Park lancé en 2000, où il est question de processus écologique, temporel et spatial, tandis que le deuxième aborde le projet « Decamping Detroit » de Charles Waldheim et de Marili Santos-Munné. Ce projet consiste à apporter une réponse à la désertion de la ville de Détroit à la suite de son déclin économique. La stratégie de (re)colonisation écologique des sites vacants et/ou abandonnés (Shannon, 2003), adoptée par les concepteurs, invite, selon Corner (2001b), à réfléchir au renversement des approches architecturales traditionnelles. Outre ce projet, plusieurs travaux théoriques et pratiques des $\mathrm{XIX}^{\mathrm{e}}$ et $\mathrm{xx}^{\mathrm{e}}$ siècles alimentent la réflexion de Corner et sa définition du Landscape Urbanism. Les approches d'Olmsted et de Jensen, le projet du parc de La Villette, les travaux de Rem Koolhaas, la pratique de West8-Adrian Geuze, etc., représentent pour Corner les prémices du Landscape Urbanism.

Dans l'article "Horizontality: Spreads and Densities in the Emergent Landscape " (2003b), Corner explique dans quelle mesure le paysage et l'écologie constituent une alternative aux modèles de plusieurs architectes et urbanistes contemporains. Des modèles qui se focalisent sur la forme urbaine et qui opposent la ville au territoire. Les enjeux des principes de l'écologie dans la pratique du projet de paysage sont mis en évidence davantage dans l'article " Not Unlike Itself : Landscape Strategy Now » (2004). L'écologie permet la compréhension des données complexes des sites, elle fournit des solutions d'organisation et d'adaptation à de grandes échelles et intervient sur un temps ouvert offrant ainsi une certaine flexibilité et souplesse aux projets. Dans cet article, Corner adopte les préoccupations fondamentales de McHarg et présente l'écologie comme un modèle stratégique répondant aux principales orientations $d u$ Landscape Urbanism.

\section{Théorie et projet (depuis 2005)}

Les années 2000 représentent l'essor de la pratique de Field Operations. Une pratique dont les débuts sont marqués par deux projets complexes, à savoir FreshKills Park et la High Line (New York). Le premier concerne la requalification de la plus grande décharge au monde tandis que le deuxième porte sur la requalification d'une voie ferrée aérienne désaffectée localisée au cœur de Manhattan. Ces deux projets peuvent être considérés comme un laboratoire pour Corner. Il expérimente et ajuste ses idées théoriques à travers ces deux projets.

Les textes de cette période reflètent ce travail itératif entre théorie et pratique. Au-delà de la présentation des projets, ils mettent en lumière des méthodes de travail 
novatrices ouvrant sur de nouvelles possibilités pour la ville contemporaine. Les deux premiers articles, à savoir : "Lifescape Fresh Kills Parkland» (2005a) et "Botanical Urbanism » (2005b), portent respectivement sur le projet de requalification de la décharge de Fresh Kills à New York et sur le projet d'aménagement du jardin botanique à l'université de Porto Rico. Pour Corner, ces deux projets ont joué un rôle important dans la définition du Landscape Urbanism. Décrit par Corner comme un essai métaphorique du Landscape Urbanism (Corner et Bick Hirsch, 2014), FreshKills Park a en partie contribué à l'évolution des principes de ce dernier (Waldheim, 2006). Quant au jardin botanique de l'université de Porto Rico, Corner le considère comme un exemple abouti du Landscape Urbanism (Corner, 2005b) car il ouvre sur une nouvelle forme de jardin botanique mettant en relation le formel et le poétique, le scientifique et l'artistique, le naturel et le culturel (figure 9).

Figure 9. Jardin botanique de l'université de Porto Rico

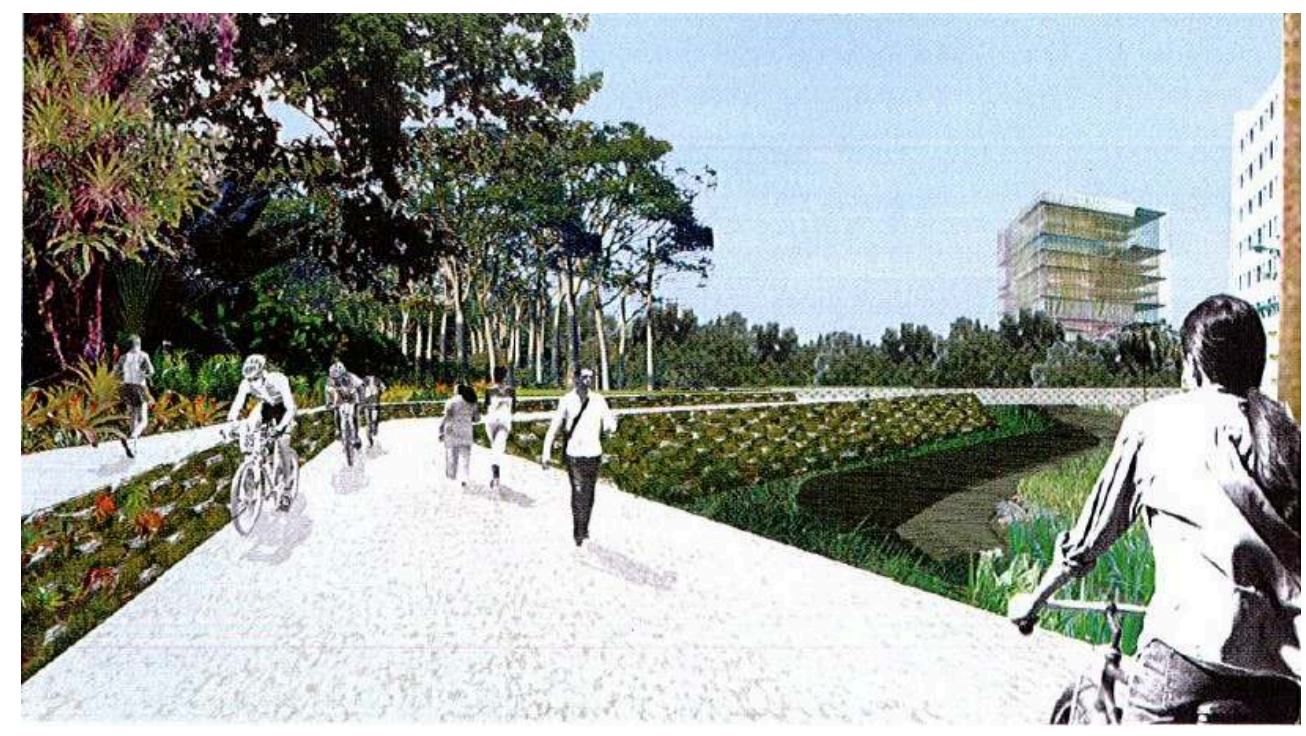

Source : Field Operations, 2004

La High Line à New York est, en outre, un projet qui a contribué à la définition du modèle opérationnel du Landscape Urbanism. Il constitue une troisième nature (au sens développé par John Dixon Hunt $^{6}$ ) qui traduit les idées de la première nature et les expériences de la seconde nature. La démarche et le processus de ce projet sont abordés respectivement dans l'article «The High Line, New York» (2009) et dans l'ouvrage The High Line, Foreseen, Unforeseen (Corner et al., 2015).

\section{Influence des travaux de James Corner sur l'émergence du Landscape urbanism}

Au milieu des années 1990, James Corner organise plusieurs événements (journées d'étude, conférences...), en collaboration avec d'autres universitaires et praticiens. L'objectif de ces manifestations est de faire évoluer les paradigmes de l'architecture du paysage (Nawre et Sen, 2011) et de développer une approche qui permet « d'explorer les paysages d'infrastructure de manière distincte des espaces pittoresques et récréatifs»(ibid., p.33). Il est important de rappeler que pendant cette période, 
plusieurs villes américaines post-fordistes sont en déclin économique. Cette situation implique divers maux urbains dont la détérioration du cadre de vie, la déshérence urbaine, la multiplication des friches, notamment industrielles, etc.

Dans ce cadre-là, Corner organise deux symposiums. Le premier, intitulé " Constructing Landscape ", s'est tenu à l'université de Pennsylvanie en 1993. Cette manifestation a réuni plusieurs théoriciens et praticiens dans le champ de l'architecture du paysage pour leurs travaux. Les participants ont interrogé le rôle du paysage dans la compréhension, la conception et la transformation de la réalité. Le second symposium, intitulé "Recovering Landscape ", s'est déroulé à l'Architectural Association (AA) de Londres en 1994. Plusieurs figures internationales de l'architecture du paysage ont été invitées à discuter et à réfléchir à de nouveaux savoir-faire pour une pratique paysagère novatrice. Une partie des contributions présentées lors de ces deux symposiums est publiée par James Corner en 1999, dans l'ouvrage collectif Recovering Landscape: Essays in Contemporary Landscape Architecture, dont l'idée générale est de promouvoir et de réorienter le projet de paysage vers des formes renouvelées plus pertinentes au regard des contextes contemporains.

Ces deux symposiums ainsi que l'ouvrage Recovering Landscape (1999a) peuvent être considérés comme à l'avant-garde du Landscape Urbanism. Ils amorcent un changement de paradigmes à travers une compréhension et une utilisation réfléchie du paysage. Un changement encadré, quelque temps après, par le Landscape Urbanism. Les articles issus de ces symposiums suggèrent le renouvellement de la pratique de l'architecture de paysage existante, notamment ses modes de représentation et son champ d'intervention. Ce renouvellement implique la reconsidération de la notion de paysage dans le projet de conception. Celui-ci est alors regardé en tant que processus plutôt qu'objet scénique, qui permet d'appréhender, à travers le temps, les caractéristiques physiques et culturelles des sites et de leurs interactions. Dans son article « Recovering Landscape as Critical Cultural Practice " introduisant l'ouvrage Recovering Landscape, Corner considère que le mot Landscape peut être (ré)interprété de manière plus fonctionnelle. Au-delà de sa considération en matière visuelle et écologique, il doit être investi en matière d'actions et de forces inhérentes, ce que Corner (1999e) désigne par "Landscape Agency ». Cette reconsidération conceptuelle couvre " un mode de relation profond et intime non seulement entre les bâtiments et les champs, mais aussi entre les modèles d'occupation, d'activité et d'espace » (Corner, 1999c, p. 154). Cette conception du paysage ouvre sur un nouveau champ des possibles, notamment dans le cadre du projet urbain.

Dans la construction du Landscape Urbanism en tant que pratique, Corner représente le premier auteur qui définit des principes opérationnels ${ }^{7}$. En 2003, dans son article "Landscape Urbanism », il propose cinq items : horizontalité, infrastructures, formes de processus, techniques et écologie, qu'il réorganise dans son article "Terra Fluxus " (2006) en quatre thèmes: processus au fil du temps, stratification des surfaces, méthode de travail et imaginaire. Cette évolution est liée à sa pratique professionnelle. En effet, pour Corner la théorie et la pratique sont intimement liées, il expérimente ses idées théoriques dans ses projets et les résultats obtenus sont théorisés, une sorte de modèle, en vue d'une réutilisation dans le champ professionnel. 


\section{Conclusion : le rôle de l'évolution de la pensée de James Corner dans l'émergence du Landscape Urbanism} examine son rôle dans l'émergence du Landscape Urbanism. Les travaux de Frederick Law Olmsted, de Jens Jensen et de Ian McHarg représentent le point de départ des réflexions de Corner sur l'enseignement et la pratique de l'architecture du paysage. Il intègre aux approches rationnelles et objectives de ses prédécesseurs une nouvelle dimension, à savoir l'imagination du paysage (Landscape Imagination). Cette dernière représente le point commun à tous ses écrits. Elle permet à la fois de comprendre et de concevoir de nouvelles formes de paysage, d'où son introduction en tant que thème clé dans le modèle opérationnel du Landscape Urbanism défini en 2006.

Par ses écrits, James Co à rner marque la pratique de l'architecture du paysage dans le temps. Il contribue à la mise en place de bases théoriques. Ensuite, il explore de nouvelles méthodes de conception et de représentation, notamment à travers le paysage et l'écologie en tant qu'Agent of Creativity. Et finalement, il contribue à la reconsidération de la définition du paysage et à l'émergence d'une nouvelle approche stratégique pour appréhender les villes contemporaines, à savoir le Landscape Urbanism. Ce renouvellement s'inspire de plusieurs projets dont le parc de La Villette de Bernard Tschumi. Le modèle opérationnel qu'il propose conjugue les préoccupations écologiques de ses prédécesseurs et sa (re)conception du paysage en tant que médium culturel complexe et processus en perpétuelle transformation. Le défi de James Corner est de réconcilier la technique et la science avec la phénoménologie et de dépasser les dualités simplistes ville/paysage, science/art, nature/culture.

\section{BIBLIOGRAPHIE}

Beard, P., « Taking Measures Across the American Landscape », AA Files, nº 30, 1995, p. 84-85.

Bélanger, P., « Everything on the Table. Reconvering and Reprojeting James Corner's Lost Map », Landscape Architecture Magazine, 2015, p. 127-132, URL : https://

landscapearchitecturemagazine.org/2015/02/10/everything-on-the-table/

Bick Hirsch, A., « Intoduction : The Landscape Imagination in Theory, Method and Action », in Corner, J., Bick Hirsch, A., The Landscape Imagination. Collected Essays of James Corner 1990-2010, New York, Princeton Architectural Press, 2014, p. 12-37.

Corner, J., Scofidio, D., Renfro, The High Line. Foreseen, Unforeseen, Phaidon, Londres, 2015, 452 p.

Corner, J., Bick Hirsch, A., The Landscape Imagination. Collected Essays of James Corner 1990-2010, New York, Princeton Architectural Press, 2014, 320 p.

Corner, J., «Preface », in Corner, J., Bick Hirsch, A., The Landscape Imagination. Collected Essays of James Corner 1990-2010, New York, Princeton Architectural Press, 2014, p. 7-11.

Projets de paysage, 24 | 2021 
Corner, J., « James Corner on Dixon Hunt », in Talarowsky, M., Weller, R., Transects. 100 Years of Landscape Architecture and Regional Planning at the School of Design of the University of Pennsylvania, San Fransisco, Applied Research + Design Publishing, 2013. p. 147-161, URL : https:// static1.squarespace.com/static/56b5181b2eeb812c64499929/t/56ba818d4c2f85c792657e0e/ $1455063440259 /$ transects+.pdf

Corner, J. Field Operations with Diller Scofidio + Renfro, « The High Line, New York, 2004-09 », Lotus international, $\mathrm{n}^{\circ} 139,2009$, p. 8-15.

Corner, J., « Terra Fluxus » in Waldheim, C., (ed.), The Landscape Urbanism Reader, New Jersey, Princeton Architectural Press, 2006, p. 23-33.

Corner, J., « Lifescape. Fresh Kills parkland », Topos: The international Review of Landscape Architecture and Urban Design, $\mathrm{n}^{\circ}$ 51, 2005a, p. 14-21.

Corner, J., « Botanical Urbanism », Studies in the History of Gardens and Designed Landscapes, $\mathrm{n}^{\circ} 2$, vol. 25, 2005b, p. 123-143.

Corner, J., « Not Unlike Itself : Landscape Strategy Now », Harvard Design Magazine, $\mathrm{n}^{\circ} 21,2004$, p. 32-34.

Corner, J., « Landscape Urbanism » in Mostafavi, M., (ed.), Landscape Urbanism : a Manuel for the Machinic Landscape, London, AA publications, 2003a, p. 58-63.

Corner, J., " Horizontality : Spread and Densities in the Emergent Landscape », Lotus International, $\mathrm{n}^{\mathrm{o}} 117$, June 2003b, p. 116-131.

Corner, J., « Downsview Park, Toronto - Emergent Ecologies », Lotus International, nº 109, 2001a, p. 52-55.

Corner, J., « Landscraping » in Daskalakis, G., Waldheim, W., Young, J., Stalking Detroit, Barcelone, Actar, 2001b, p. 122-126.

Corner, J., Recovering Landscape : Essays in Contemporary Landscape Architecture, New York, Princeton Architectural Press, 1999a, 288 p.

Corner, J., « Field Operations », Architectural Design, vol. 69, n 7/8, 1999b, p. 52-55.

Corner, J., « Eidetic Operations and New Landscapes ", in Corner, J., Recovering Landscape : Essays in Contemporary Landscape Architecture, New York, Princeton Architectural Press, 1999c, p. 153-169.

Corner, J., «The Agency of Mapping. Speculation, Critique and Invention », in Cosgrove, D. (ed.), Mappings, London, Reaktion Books, 1999d, p. 188-225.

Corner, J., «Introduction : Recovering Landscape as a Critical Cultural Practice », in Corner, J., Recovering Landscape : Essays in Contemporary Landscape Architecture, New York, Princeton Architectural Press, 1999e, p. 1-26.

Corner, J. « Ecology and Landscape as Agent of Creativity », in Thompson, G., Steiner, F., (eds.), Ecological Design and Planning, New York, John Wiley \& Sons, 1997, p. 80-108.

Corner, J., MacLean, A., Van Valkenburgh, M., Taking Measures across the American Landscape, New Haven, Yale University Press, 1996, 185 p.

Corner, J., « Representation and Landscape : Drawing and Making in the Landscape Medium », Word \& Image, vol. 8, n 3, 1992, p. 245.

Corner, J., " A discourse on theory II : “Three Tyrannies of Contemporary Theory and the Alternative of Hermeneutics" ", Landscape journal, n 10, 1991, p. 115-133. 
Corner, J., « A discourse on theory I : "Sounding the Depths" - Origins, Theory, and Representation », Landscape journal, n 9, 1990, p. 61-78.

Donadieu, P., « Landscape Urbanism in Europe : from Brownfields to Sustainable Urban Development », Journal of Landscape Architecture, JoLA, vol. 2, n 2, 2006, p. 36-45.

Drake, C. S., « Term, Definitions, Identity », Topos. The international Review of Landscape Architecture and Urban Design, $\mathrm{n}^{\circ}$ 71, 2010, p. 50-57.

Duany, A., Talen, E. (dir.), Landscape Urbanism and its Discontents. Dissimulating the Sustainable City, Gabriola Island, New Society Publishsers, 2013, 336 p.

Field Operations, «Fresh Kills Park: Lifescape. Draft Master Plan », 2006, URL : https:// freshkillspark.org/wp-content/uploads/2013/07/Fresh-Kills-Park-Draft-Master-Plan.pdf.

Hunt, D., « Les Promenades de Paris et le Landscape Urbanism », dans Audouy, M., Le Dantec, J-P., Nussaume, Y., Santini, C. (dir.), Le Grand Paris[s] d'Alphand. Création et transmission d'un paysage urbain, Paris, Éditions de La Villette, 2018. p. 139-146.

Leger-Smith, F.-A., «Évolution des pratiques des paysagistes face aux enjeux écologiques de la conception urbaine ", thèse de doctorat sous la direction de W. Oueslati et H. Davodeau, Agrocampus-Université d'Angers, 2014. 288 p.

McHarg, I., Design with Nature, New York, Falcon press, 1969, 198 p.

Mostafavi, M., (ed.), Landscape Urbanism : a Manual for the Machinic Landscape, London, AA publications, 2003, $176 \mathrm{p}$.

Nawre, A, Sen, S., «In conversation with Charles Waldheim and James Corner », Journal of Landscape Architecture, $\mathrm{n}^{\circ}$ 31, 2011, p. 32-37.

Shannon, K., « Stalking Detroit by Georgia Daskalakis, Charles Waldheim, Jason Young », Landscape Journal, $\mathrm{n}^{\circ}$ 1, vol. 22, 2003, p. 67-69.

Steiner, F., « Landscape Ecological Urbanism : Origins and Trajectories », Landscape and Urban Planning, $\mathrm{n}^{\circ}$ 4, vol. 100, 2011, p. 333-337.

Talarowsky, M., Weller, R., Transects. 100 Years of Landscape Architecture and Regional Planning at the School of Design of the University of Pennsylvania, San Fransisco, Applied Research + Design Publishing, 2013, 230 p., URL : https://static1.squarespace.com/static/ 56b5181b2eeb812c64499929/t/56ba818d4c2f85c792657e0e/1455063440259/transects+.pdf Thompson, I. H., « Ten Tenets and Six Questions for Landscape Urbanism », Landscape Research, vol. 1, n 37,2012 , p. 7-26.

Van Valkenburgh, M., « Foreword », in Corner, J., MacLean, A., Van Valkenburgh, M., Taking Measures across the American Landscape, New Haven, Yale University Press, 1996, 185 p.

Vicenzotti, V., « The Landscape of Landscape Urbanism », Landscape Journal, $\mathrm{n}^{\circ}$ 1, vol. 36, 2017, p. 75-86.

Waldheim, C., (ed.), The Landscape Urbanism Reader, New Jersey, Princeton Architectural Press, 2006. $288 \mathrm{p}$.

Weller, R., «Introduction », in Talarowsky, M., Weller, R., Transects. 100 Years of Landscape Architecture and Regional Planning at the School of Design of the University of Pennsylvania, San Fransisco, Applied Research + Design Publishing, 2013, p. 7-11, URL : https:// static1.squarespace.com/static/56b5181b2eeb812c64499929/t/56ba818d4c2f85c792657e0e/ $1455063440259 /$ transects+.pdf 


\section{Webographie}

Garlic Idealab, James Corner Interview, Youtube [11' 43"], 22 September 2017, URL : https:// www.youtube.com/watch ?v =PJ4nfzsaVhI

\section{NOTES}

1. Un certificat d'honneur et de mérite attribué aux étudiants diplômés en architecture du paysage dont les travaux démontrent un potentiel de contribution exceptionnel à la profession.

2. Connue initialement comme l'agence Wallace \& McHarg Associates, elle évolue en 1963 avec l'arrivée dans l'équipe dirigeante des deux architectes paysagistes William Roberts et Thomas Todd. En 1979, Ian McHarg quitte l'agence.

3. Architecte paysagiste, elle a dirigé (1986-1994) et enseigné (1986-2000) au département d'architecture et de paysage de l'université de Pennsylvanie.

4. Un tableau horizontal constitué de plusieurs films d'information transparents superposés, comprenant cartes, diagrammes, dessins, photos et textes. Ce tableau est conçu comme une table autour de laquelle les acteurs du projet peuvent discuter de l'avenir de leur ville.

5. Créé par un groupe d'hommes politiques et de scientifiques du Massachusetts Institue of Technology (MIT), le club de Rome s'est fait connaître au-delà des USA avec la publication en 1972 du rapport The Limits toogGowth. Ce rapport examine et analyse l'impact écologique de la croissance économique et démographique des Trente Glorieuses.

6. Selon la catégorisation de Dixon Hunt : $1^{\text {re }}$ nature correspond au sauvage (wildness), la $2^{\mathrm{e}}$ nature implique les paysages issus de l'activité agricole primaire et la $3^{\mathrm{e}}$ nature est induite de la pratique des jardins.

7. Quelques années plus tard Ian Thompson (2012) propose dix principes du Landscpae Urbanism.

\section{RÉSUMÉS}

James Corner, paysagiste américain contemporain, a largement contribué au développement et au renouvellement du champ de l'architecture du paysage aux États-Unis. Sa pensée et ses projets s'inscrivent dans la continuité des idées de Frederick Law Olmsted (1822-1903), de Jens Jensen (1860-1951) et de Ian McHarg (1920-2001), des figures emblématiques qui ont marqué un tournant dans la pratique du projet de paysage. Dès le début des années 1990, James Corner mène des travaux portant sur la théorie et la pratique du projet de paysage. Ces travaux s'inscrivent dans un contexte de renouvellement de paradigmes; ils (ré)interrogent, au prisme des enjeux urbains contemporains, les fondements théoriques, les outils et la démarche du projet de paysage. À la fin des années 1990, James Corner contribue, avec les architectes Charles Waldheim et Mohsen Mostafavi, à la définition et au développement du Landscape Urbanism en tant qu'approche novatrice. Par l'analyse des écrits de James Corner, cet article met en lumière l'évolution de sa pensée théorique dans le temps et examine le rôle de cette évolution dans l'émergence du Landscape Urbanism.

The contemporary American landscape architect James Corner has made a major contribution to the development and renewal of landscape architecture in the United States. His approach and 
his projects are in line with the ideas of Frederick Law Olmsted (1822-1903), Jens Jensen (1860-1951) and Ian McHarg (1920-2001), emblematic figures who marked a turning point in the practice of landscape architecture. Since the beginning of the 1990s, James Corner has been working on the theory and practice of landscape architecture. This work is part of a paradigm shift ; it challenges, in the light of contemporary urban issues, the theoretical foundations, tools, and approaches of landscape architecture. At the end of the 1990s, James Corner contributed, along with architects Charles Waldheim and Mohsen Mostafavi, to defining and developing the concept of Landscape Urbanism as an innovative approach. Through an analysis of James Corner's writings, this article highlights the evolution of his theory over time and examines the role it has played in the emergence of Landscape Urbanism.

\section{INDEX}

Mots-clés : James Corner, théorie de l'architecture du paysage, pratique du projet, représentation, Landscape Urbanism

Keywords : James Corner, theory of landscape architecture, the practice of landscape architecture, representation, Landscape Urbanism

\section{AUTEUR}

\section{IMÈNE OUALI-BOURAHLA}

Imène Ouali-Bourahla est architecte doctorante, École doctorale de géographie (ED 434), université Paris 1 Panthéon-Sorbonne, unité de recherche Architecture, Milieu, Paysage (AMP), École nationale supérieure d'architecture Paris La Villette (ENSAPLV).

ouali.imene[at]gmail[dot]com 